\title{
RTS/CTS Framework Paradigm and WLAN Qos Provisioning Methods
}

\author{
Mohamed $\mathrm{Nj}$. \\ Dept. Computer Communications \& Network Systems \\ FTMK - Teknikal Universiti Malaysia \\ Melaka, Malaysia
}

\author{
S. Sahib \\ Dept. Computer Communications \& Network Systems \\ FTMK - Teknikal Universiti Malaysia \\ Melaka, Malaysia
}

\author{
N. Suryana \\ Dept. Software Engineering \\ FTMK - Teknikal Universiti Malaysia \\ Melaka, Malaysia \\ B. Hussin \\ Dept. Industrial Computing \\ FTMK - Teknikal Universiti Malaysia \\ Melaka, Malaysia
}

\begin{abstract}
Wireless local area network (WLAN) communications performance design and management have evolved a lot to be where they are today. They went through some technology's amendments and innovations. But, some performance tools remained almost unchanged and play a fundamental role in contemporary networking solutions despite the latest innovations higher influence on their indisputable and important function. That is the case with Request to send (RTS) and consent to receive (CTS) protocols. They are among the former technologies, which helped for transmission control with better performance in WLAN environment. They are so important, particularly since the advent of sensitive data networking (e.g. internet telephony, audio and video materials distribution) over the internet protocol (IP). Up to recent years following today's multimedia WLAN based networks deployment trends, RTS/CTS) contributed to provide networks with some expected good performance levels prior to the discovery of more sophisticated methods for this purpose (i.e. performance enhancements). And yet, one may question whether the new technologies have rendered RTS/CTS frameworks obsolete; or are they now used only for some specific network applications traffic management? This articles review attempts to comprehensibly study some of the research works, which have had interest in RTS/CTS mechanism as tools for WLAN applications performance support. Various researches have studied these tools from their early innovation as network node's built-in component, through different frameworks associated with WLAN legacy (IEEE 802.11) MAC protocols. This paper analyzed RTS/CTS initial implementation as mere network performance solution from packets' collision avoidance perspective; and then for transmission delay due to hidden nodes and their false deployment. The article closes up on a critical analysis on the possible long time contribution of these protocols into integrated schemes based WLAN QoS performance design.
\end{abstract}

Keywords-RTS/CTS; MAC; Internet; Telephony; video; realtime; loss; multimedia; WLAN; mechanism; performance; protocols, collision; framework; transmission; reception; flow control; handshake; MANET; BSS; IBSS; QoS

\section{INTRODUCTION}

Request to send and consent to receive (RTS/CTS) are one of the main elements of flow control on network communicating nodes, which acts at such individual device level as a special gateway for data transmission (Sending and reception). They are sockets/ports embedded into almost every network's end-nodes. Their mechanism's function is very important and thus more valuable on the client side performance's management. In fact, many research works have been done about local area network (LAN) and wireless LAN (WLAN) management using RTS/CTS as performance support to their different service applications. WLAN is an ever great platform tool for wired networks (LAN) extension to wireless and then mobile networks of all kinds. RTS/CTS usefulness is reported in various studies. First, they are a feature of the IEEE 802.11 WLAN technology having initially for main function to control station access to the shared medium. A correct implementation (Timing on/off and threshold setting) of RTS/CTS lets user adjust/regulate the WLAN packets transmission relatively to the operation environment [1]. Practically, these sockets on the client's device enable timing packets transmission after making first a request (RTS frame sent) and receiving reply (CTS frame received) from a peer network node $[2,3]$. They are used as an optional technique [4] in wireless LAN legacy (IEEE 802.11) for transmission control between clients and the access-point (AP) $[2,4]$; they are so known also as best tools in negotiating or ensuring bandwidth $[2,4,6]$ prior for a client transmitting its data.

In WLAN environment, radio interference can occur relatively to the terminals' location and position to the AP; this is generally known as hidden terminal issue [3, 4]. Further details to this issue are under sections three and four of this article. A systematic consequence of this situation is the packets collision at the AP for attempted transmissions between any client and the hidden one. However, collision is proven in literatures as the basic source for data loss. In turn, this will lead to end-to-end (E2E) throughputs decrease as negative effects with undesirable delays (e.g. in phone calls). To ensure WLAN good performance, IEEE 802.11 media access control (MAC) uses either of the following two techniques against the interference occurrence: RTS/CTS mechanism and the physical carrier sensing media access 
(CSMA). RTS/CTS handshake is a virtual carrier sensing known as perfectly able to reduce interference and related consequences $[2,3,4,5]$. RTS/CTS thresholds proper settings along with a good adjustment of wireless local area network (WLAN)'s clients within the access-point (AP) transmission range are among the strategic methods to obtain good control for great performance of the network [2, 3, 4].

Comparing the two above mentioned techniques, the second is proven less effective in solving for the interference issues in WLAN. In fact, this remark is made relatively to the RTS/CTS potential capability for the same task of controlling clients transmission to avoid collision occurrence [6]. In other words, RTS/CTS efficiency is subject to the use with each of WLAN operation modes (i.e. Basic set of services (BSS) and Independent BSS (IBSS)). Despite the wireless technologies development for different application's perspectives, yet the above stated matter still holds much believe for RTS/CTS support capability. For instance, in sensor wireless network environment (IBSS-WLAN) the use of geographic positioning system (GPS) as part of integrated solutions support sounds only very advanced hints, but limited in efficiency. Overall, attempted solutions can be tried and only manually [7]. In general, WLAN contemporary deployment method allows users exploring flexibly the networks, including telecommunications network operators to rate by the wireless users $[8,9]$. However, the typical ways of deploying WLAN for a same purpose or more include (a) LAN extension, (b) cross-building interconnect, (c) nomadic access, and (d) adhoc networking [10]. Despite their performances solution differences, they experience hidden stations or nodes localization and position issue. The deployment objectives and advantages out of various studies' review show almost the same findings [11]. Namely as LAN extender and enabler for users mobility when connected; currently the best of internet gateways (Portal) whether indoor or outdoor use due to many related benefits: cheap cost and easy deployment for requiring only little IT-knowledge for systems configuration; moves with and access to various applications and services regardless of server location and time; etc. Wireless operations made it possible short and long distance communications, including the unrealizable projects in wired networking [12]. Hence, such a great evolution in communications technology has allowed a vast majority of little and even zero-level computer literacy people to get exposed to both the advance telecommunication system (e.g. Internet, IP based telephony and smart-telephony) and wireless mobile networking (e.g. mobile WLAN, smartphone uses). This group of consumers in fact makes up a considerable number of consumers in internet and telecoms market especially in third world countries. Hence, this situation calls upon the industry and service providers (NSP) attention in providing them with more easier means for network troubleshooting during their access to network.

The foremost and particular learning from above sources and related studies was/is about the importance of wireless networks performance management using lower level tools to support possible automated solutions from all other network's levels. This article attempts to highlight the lack of enough discussions on such provisions in QoS or performance based new research papers. This remark includes a limited access offer to those tools' features, with RTS/CTS threshold settings as special case of concern [33, 34]. Some recent QoS surveys based articles have helped much verify this remark, as analysed here in sections three and four.

\section{WLAN OPERATION AND PERFORMANCE ISSUES}

Request to send and consent to receive (RTS/CTS) mechanism is among the tools in early network technology embedded on networking communications hardware. WLAN standard IEEE 802.11 contains RTS/CTS protocols to control clients access to shared-medium according to configured threshold [5, 6]. In fact, WLAN for open access/hotspot (e.g. Cafés, Offices, Hostel, etc.) faces multiple users at various locations/positions. Thus, in such complex situations, traditional equipment is merely inadequate to fairly deal with the rate of clients' service demands and generated interferences. However, some friendly management tools are available but only on expensive products. They provide users with RTS threshold settings in WLAN radio network interface cards (NIC) and AP interfaces $[3,6,5]$.

\section{A. WLAN Basic Operation Modes Overview}

Wireless LAN is obviously considered as the base network for modern mobile networks access in home, office and medium organization's services.

\section{1) WLAN Types of Deployment}

The legacy (IEEE 802.11) provides two operation modes. There is infrastructure mode: - in which wireless stations remain in mobile communications but depend strictly on the AP radio coverage range, which act as bridge to other subnets, LAN and the internet. And the other is ad hoc mode: - for which mobile wireless stations interact directly in a peer-topeer manner. In both operation modes, WLAN MAC protocols configurations for physical layers and medium access are the same, despite some modifications in 'mobile adhoc network' (MANET) case [13, 14]. However, MANET systems experience different topologies relatively to the mobile nodes location and positions to each other over time. Thus, such a change situation is a cause to their operation problems and solutions difference [13]. Hence, various research studies showed that RTS/CTS mechanism is not considered suitable for MANET environment but instead CSMA/CA based distributed MAC in each terminal $[5,6,15]$.

\section{2) Mobile Ad-hoc Network}

Mobile ad-hoc networks (MANET) known also as independent basic set of services (IBSS) based WLANs are the model suitable in the regions impracticable for ordinary wired network deployment. In MANET, mobile stations are self- networks deployment [14]. In MANET, mobile stations are self-organized [13, 14] and therefore they need a distributed MAC. In fact, shared MAC or shared bandwidth mechanism is more convenient in infrastructure based WLANs [13]. The solution scheme for hidden stations/collisions in MANET preferably [13] use the called 'carrier sense multiple access with collision avoidance' (CSMA/CA). As a lesson from above references, RTS/CTS successful use requires WLAN manager's attention for proper additional control and necessary adjustments. Hence, since 
MANET system does not possess such centralized management, therefore CSMA/CA and variant mechanisms make senses as choice for applicable solutions scheme in such type of wireless networks.

\section{3) WLAN'S Clients Communications Issues}

Whether in infrastructure based or in MANET operationmode, WLAN faces either of the following performance challenges: (a) bandwidth limitation, (b) radio interferences, (c) transmissions collision, (d) congestion, and (e) outcome problem. Each of these problems has a critical impact on the WLAN applications performance, especially on the VoIP applications (e.g. Calls, videos, and other real-time transactions application). In general, interferences can cause packets collisions due to hidden receiver-station (Fig. 1) leading to E2E delivery decrease. In the other hand collisions induce congestion, which cause throughputs reduction and more in delay. The increase in delivery delay originates from retransmissions and its execution time $[3,4,6]$. In overall, the network operation ends up with a poor services quality resulting from performance degradation due to the above stated facts $[6,15]$.

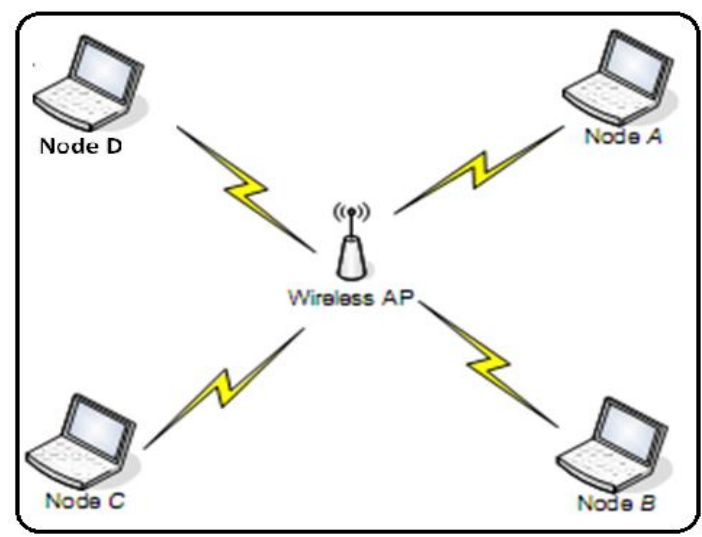

Fig. 1. WLAN hidden 'receiving' terminal issue illustration

However, in wireless network, hidden receiving stations problem is among the first severe causes of WN performance degradations [13] and thus low QoS [3, 4]. Technically wireless network's invisible and exposed nodes problem is generally due to either radio wave interferences (e.g. Case with huge of users in hotspots and condominiums), or short range between contending nodes [6, 13, 15]. Therefore, network topology must be accounted at least in maintenance process; that is because of the position nodes location involvement into hidden nodes problem. In fact, the use of RTS/CTS mechanism helps fairly tackle the unheard or hidden station's issue. So doing, this strategic solution leads in turn to another similar problem known as exposed nodes problems [4, 14]. That is, once any of the exposed nodes (e.g. Fig.1 'A' node) hears/senses the CTS exchange originated from a station (e.g. Fig.1 node ' $C$ ') to which it wanted also to transmit packets to, this node ('A') will simply drop its own packets without genuine proof for probability of loss [14] in the case that those packets could have been sent. And, such false abstentions consequently will gradually reduce the network throughputs delivery [2-6, 15], then the performance and finally the QoS on top of all.
The technical causes to hidden receiving node are discussed under solution's design (section IV). However, the scenario portraying unheard terminal can be illustrated like in Figure 1. Any of the four stations can fail to connect with either of three others though they are logically interconnected through a same Wireless AP (WAP). There are almost three general cases with this problem as discussed in [15]: (a) either all nodes cannot hear each other; (b) - or they are visible but in contention for resources each other; (c) - and else they are invisible stations and contention happens simultaneously.

As particular learning, "many past researches have proved that about $40 \%$ of packets loss in wireless networks occurred because of invisible terminals problem" [15]. Therefore, welldesigned RTS/CTS threshold and its proper implementation remain the fundamental way to solve the issue and incumbent defects $[1-6,15,35]$. The analyses in recent studies among these sources are implicitly an alert for more consideration to this solutions framework due to some observed achievements. On the IEEE 802.11 physical carrier sensing, some alternative methods (e.g. Clear channel assessment (CCA), fragmentation, queuing discipline, etc.) to RTS/CTS have been explored and tested for comparison between their potential extents [36-38]. But, RTS/CTS still over performed and better promised more hopes for services applications requiring QoS [39, 40] .Thus, multimedia based network applications can expect more for great QoS performance supported by integrated solutions. This is understandable, since local network managers need to enable/disable RTS/CTS assignments where and when applicable. It is then a coordinated effort to maintain performance in addition to any QoS level obtained from the network service provider. Furthermore, such above highly rated and pertinent remark from many studies is significant enough to prove that RTS/CTS fundamentally are needed in support to any other single or multiple schemes based performance solutions.

\section{B. How Does RTS/CTS Mechanism Operate?}

RTS/CTS are another alternative WLAN MAC operations support [3,6], which can be manually configure as a typical solution against wireless network (WN) frame exchange collisions [1-6, 15]. This mechanism comes into playing its function by enabling /disabling its thresholds based on the WN behaviour with respect to the throughputs decrease level as observed by the network managers $[13,14]$. However, its inadequate settings can degenerate instead into the network performance failure if not implemented based on proper finding out of the WLAN behavior's survey and results analysis. The use of thresholds must then according to findings.

\section{a) Application of RTS/CTS Mechanism}

Functionally, in shared MAC medium, RTS/CTS mechanism enables controlling the WLAN client's frames exchange with others clients within same or outside subnets via the AP. The protocols handshake's algorithm (Table 1) uses one of the following control techniques - carrier sense multiple access with collisions detection (CSMA/CD), or carrier sense multiple access with collision avoidance (CSMA/CA) mechanism [5]. To enable or disable RTS/CTS mechanism means to 'activate/deactivate' its protocols 
system. In practice, this technically has to do with the action of 'configuring the thresholds. These are parameter's values that will decide and model the behavior of packets during their transmission process. Moreover, for many literatures, these control settings are performed on the wireless clients; for, these are either a source or an end node of the packets transmission $[2,6]$. The thresholds are not applied on the AP $[2,3,4]$. Systematically, AP intervenes instead as a referee and dispatcher of frames between sources and destinations. While this control mechanism's configuration interests more the client side $[4,5,6]$, the AP by default learns from the clients operation. Then, to play its own role the AP quickly adapts to the clients traffic behavior, which depends on the applied RTS/CTS thresholds [2, 3].

\section{RTS Enabled versus Disabled:}

When a terminal's RST/CTS are activated, it always holds down its packets from sending and will release them only after obtaining CTS frame from intended destination (Figure 2.2(a)). Thus, this process enables minimizing packets collisions occurrence and thus improving the network performance [2, 6]; see Figure 2 and Table 1.

\section{RTS Disabled}

When RTS is disabled on a terminal (e.g. AP, endnode/client) this latter relies on the WLAN MAC technique called physical carrier sensing (PCS) for that terminal's packets transmission's control [2, 3, 4, 6]. However, clear channel assessment (CCA) mechanism makes used of PCS threshold to check and decide for which among nodes contending for channel free can safely transmit. Thus many literatures [14, 36,41, 42] claimed for the efficiency of this method in fairly handling the hidden stations. In addition, almost all research studies on wireless ad-hoc networks favor the used of PCS versus RTS/CTS mechanism as solution to combat collisions occurrence.

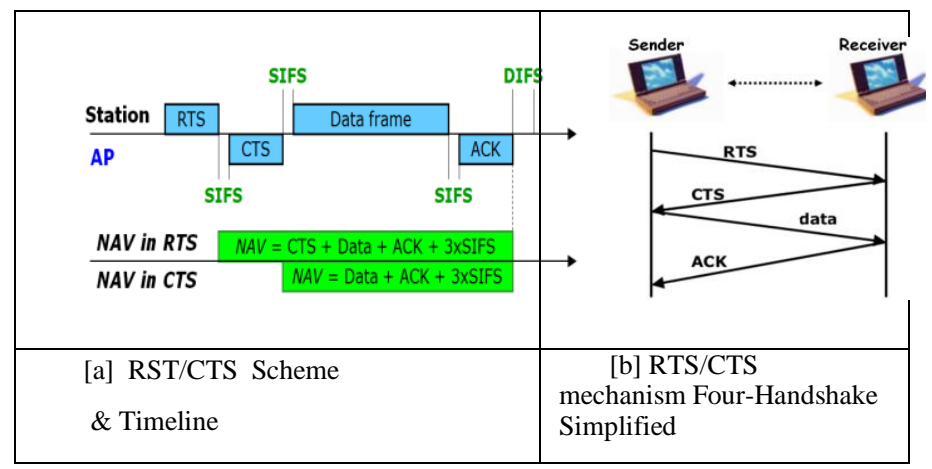

Fig. 2. RTS/CTS scheme in BSS based WLAN
TABLE I. BSS HANDSHAKE ALGORITHM (FIGURE 2.B)

1) 'A' ready to send frame to 'B': All stations' waiting period/Initial $\rightarrow$ DIFS

2) 'A' ready \& send request (RTS) to 'B' (via AP, controls and dispatching point)

3) $\mathrm{AP}$ (Intercepts A's request) issues CTS to all others (C, \& D) with timeout

4) 'B' (responds via AP) sends frame 'CTS' to 'A'

5) ' $A$ ' (Packets) send data to ' $B$ ' (via AP)

6) 'B' (ACK frame) send ACK to 'A' (via AP)

b) Protocols RTS/CTS Operation Explanations

Case for Shared MAC and Exclusive Single Access:

This refers to WLANs operating in BSS mode. The label "single exclusive access" $[3,6,15]$ indicates the fact that one and only one station's packets can be transmitted once the AP has declared the medium idle. That is clear since in this case the interactions between clients rely on the AP MAC ruling, contrary to MANET where everyone owns its MAC and BSS, despite of sharing open wireless network.

Handshake in BSS (Fig. 1\&2):

This scenario has 4 terminals (A, B, C \&D). Say, " $A$ " is ready to communicate with " $\mathrm{B}$ ". Thus, " $\mathrm{A}$ " first initiates it with a request (frame RST) via the AP. Then, AP reacts to this request with a CTS frame to all [2]; but, C \& D will receive it along with timeout value (10-16 $\mu$ seconds) [16] as "warning" about medium busy (i.e. they must hold-down their request if any for this period). Thus, "A" can release its frame toward "B" upon receiving the CTS. And then the session closes up with ("B")'s acknowledgment (ACK) frame back to "A".

For every BSS client, Figure (2.a) shows that RTS/CTS scheme relies mainly on both short inter-frame space (SIFS) and the network allocation Vector (NAV) for wireless medium access management $[2,3,15,17,18]$. SIFS controls the time interval (SIFS $=10$ to $16 \mu \mathrm{s}$ ) between consecutive frames crossing the shared medium. NAV assures (up to 50 $\mu$ s, longer enough) [16] free medium use only for sender-receiver exchange of frames. Finally, the next frame (RTS) from one of two among stations initially put on queue will be the station having just for SIFS timeout; the one with DIFS timing will be set back on queue [17].

As learnt lesson, one can analyze these conditions associated with RTS issuing, receiving CTS before releasing packets and then ACK to packet's sender ending a session. They are the proven facts on the efficiency of this mechanism against the collisions occurrence. Therefore, RTS/CTS 
handshaking offers enough control on the shared medium access $[3,6,13,15,16,17]$. Various research studies warn about the unnecessary implementation of RTS/CTS. For, this can create the called "induce congestion", resulting into some increase in overhead and thus a network performance dropping.

\section{Case for Multiple Accesses MAC:}

The reference [15] presented an example of handshake using multiple accesses with collision avoidance for Wireless (MACAW) technique. The packet transmission control in the MACAW is similar to the case discussed in Figure 2. But, MACAW applies instead a pattern of RTS/CTS/Data Sending (DATA/ACK / (DS)) for data transmission. A detailed presentation of these solutions is available in [15] study article.

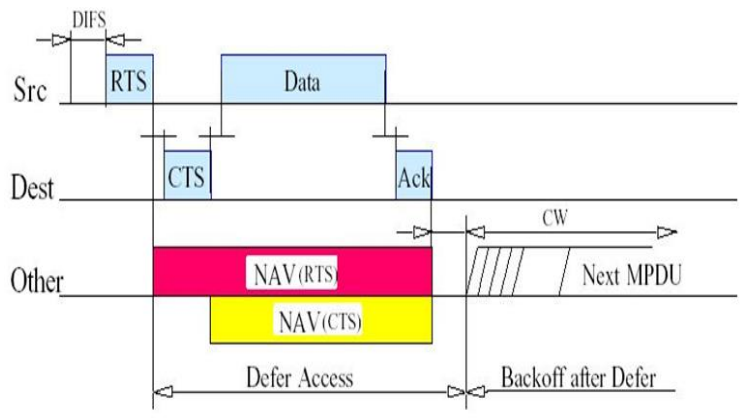

RTS/CTS Handshake Random Access MAC protocols for MANET support

Fig. 3. RTS/CTS scheme in IBSS based WLAN or MANET

RTS/CTS handshake random access MAC protocols (Figure 3 ) are the scheme model suitably applicable on mobile stations in wireless ad-hoc network [16, 17, 18, 19]. Since every mobile station owns a MAC, the access to the medium is individually negotiated; thus random access, because of various attempts based on the back-off space and differ access behaviors. The references $[14,15,16,18,19,20,21]$ discuss more in-depth about this scheme operations.

\section{c) Managing RTS Induced and Normal Congestions}

RTS induced congestion is linked to WLAN MAC layer operation. However, normal or systematic congestion happens on the TCP/IP based networks transmission as a result of buffers' overflow [6]. Otherwise, a network overall congestion situations can be considered (Figure 4) as an accumulation of these two [2,6]; but right at those moments, the induced component is so light and instantaneously last to be really accounted.

\section{d) End-to-End Throughput Theoretical Model}

The following graph in (Figure 4) shows the theoretical performance of WLAN or mobile wireless network. The performance degradation based congestions does not reach the core networks. From the literatures, congestion can be broadly viewed as the networks performance degradation origin. Another most important lesson is the great impact on the E2E caused by congestions relatively to the network loads increase (Figure 4). This remark shows that the good design and management of local network hooked to the internet would contribute to networks high performance.

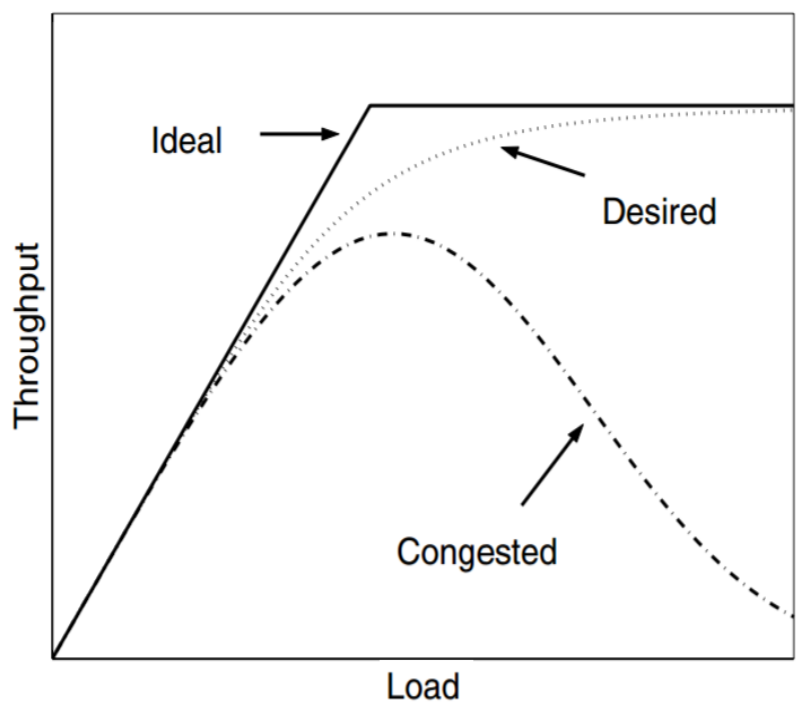

Fig. 4. WLAN theoretical performance graph showing the degradation

(E2E decrease) during congested periods [6]

Figure 5 displays the theoretical curve for network congestion in terms of the main influencing factor - network load.

\section{Congestion Control Categories}

With reference to Figure 4, the following diagram in Figure 5 shows the congestion controls commonly applied methods. In findings, all the reviewed papers showed that congestion happens actually at the open networks level. It occurs typically between different subnets as a result of a probable poor control at LAN/WLAN management level. Therefore, starting at local network's clients stage, RTS/CTS have a considerable role to play along with other associated tools for performance management at networks level.

Here are some practical solution methods to handle induced and normal congestion.

- RTS/CTS induced congestion can be cut-down by controlling and manually modifying the RTS applied threshold (e.g. packets size) [2, 3. 4. 6]. In fact, various new features supporting RTS/CTS operations make it possible to sharply minimize induced congestion occurrence - e.g. MACAW, which include an ACK at the WLAN MAC level [6].

- LAN/WLAN normal or systematic congestions can expand beyond subnet's section via the interconnection-points and cause the open networks congestion. However, a set of mechanisms is available for this level of congestion management. For examples Detection and avoidance; control detection and removal when already occurred [22].

Figure 5 displays the congestion control categories and their management relevant policy tools. The implementation decision depends on the problem model and particularly the solution type and scheme (i.e. simple/single mechanism; multiple mechanisms and thus integrated solutions). 


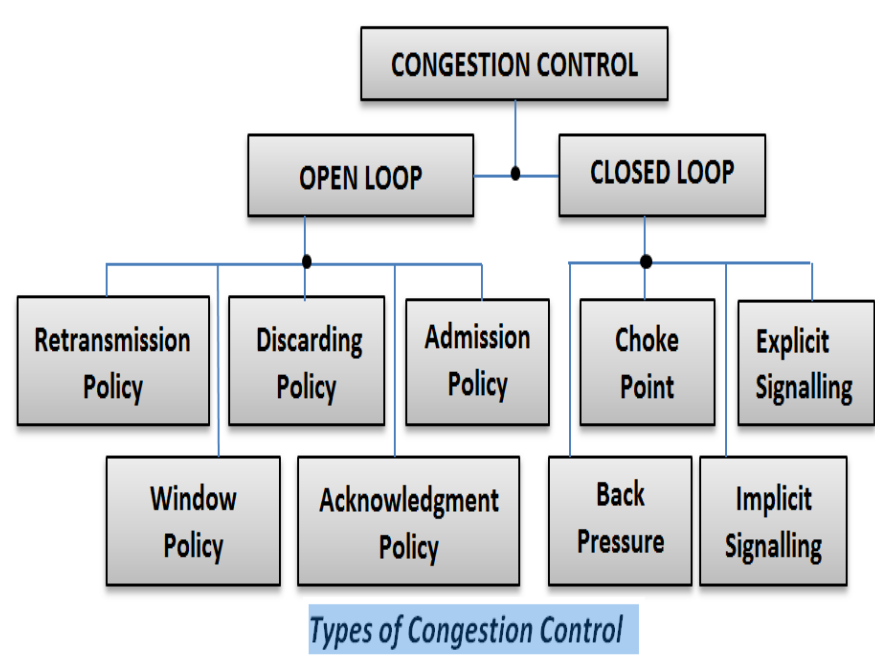

Fig. 5. Congestion controls Common Methods -- the two congestion control categories and their respective policy members - Adapted from [22]

\section{PERFORMANCE AND QOS BACKGROUND OF WIRED AND WIRELESS NETWORKS}

In performance and QoS survey papers, WLAN operation faces mainly multiple technical challenges $[12,22]$ but, just a huge of efforts are deployed on typical issues over time by the industries and vendors for solutions support or with new systems/products' added features. However, QoS by definition is expected to primarily target user's satisfaction. This would include other satisfaction's aspects like services cost, user (data and personal info) security, mobility and network availability whenever needed, etc. Most of these factors are engineering based tasks. Therefore, the most important can be those allowing users to enjoy the networks use. That will be then a result of a good job done by remote and local solutions support. In general (if not in most cases) users need some immediate and friendly methods/tools (e.g. Simple and direct troubleshooting guides on common surfing issues) for quick help; and that would be good enough to their satisfaction.

What are the listed performance/QoS issues and solution methods in recent survey papers including [11, 23, 24, 31] articles (and unread ones)? And then, what have been proposed for user's emergency basic tools? In findings from reviewed ones, there is little in offer (explanations); whereas much is being said also but, more are in technical ways. And at practical level, possible helpful details (settings) are available only on expensive products (e.g. routers and wireless router (AP) and some end-nodes - workstation, laptops and smart phones among shared systems). Therefore, only WN/WLAN managements generally are able to take required actions when a problem arises. The approach of this papers review is more about the absence of such details in recent study's discussions, which can be a valuable input, a reminder to the networks people on the matter.

\section{- Performance and QoS Meaning Confusion Impact}

In computing, the word 'performance' has two interpretations: (a) --a computer operation's speed by counting operations or instructions executed, (b) -a computer system outcome in term of "throughput" (i.e. E2E packets \# sent or received), node's response time, and availability [25]. Quality has to do with a standard of something considered against (or relatively to) many others of same kind based on its degree of excellence. Such standard is actually hard in wireless network or technology things but achieved generally by relativity to the nearer consensus of people. According to Margaret Rouse (2006) [26], "in information technology product or service, quality is sometimes defined as meeting the requirements of the customer". Thus, the networks QoS focuses on user's opinion (satisfaction) for such standard definition.

Performance and QoS are then better understood mainly from their practical interpretations at the end-users level, which are the LAN/WLAN's clients where application services' outcomes are visualized and thus appreciated. A comprehensible demo with grade of services $(\mathrm{GoS})$ versus QoS is available in the [27] article. Similarly, the structure of a QoS operation from the network to the client level shows that the process is basically about a coordinating activity from different network sections out of which the outcome can be displayed at every LAN/WN's client.

WLAN QoS faces some major technical challenges as illustrated in $[28,29]$. Based on the literatures including these above references and particularly [27], network user's experience is much influenced by the cost and marketing of the services (i.e. NSP). Thus, their satisfaction from received services is a mix of non-technical and technical facts. However, their agreement for the QoS relies particularly on the technical result experienced on their terminal (regardless of what has been said much in marketing or selling prices). Their quality of experience (QoE) encompasses their expectation) and facility or system use. Thus, any possible discrepancy or mismatches in their hopes can merely degenerate into discomfort and poor feelings about the QoS [27]. Therefore, if all the great technical works are done for the QoS at the network layer only, it is likely to not actually reach the main targeted objective, which is the customers' satisfaction. They can be offered more friendly use tools to face WN's common issues whenever necessary during networks access. For learning and guidance to solution designers, [29] article introduced about some necessary understanding of the QoS mechanisms as defined today in the IEEE 802.11; and unfortunately, RTS/CTS was not addresses into that well-summarized materials.

\section{WLAN PERFORMANCE USING RTS/CTS FRAMEWORK}

In general, there are two optional transmission control methods within WLAN environment - the use of physical carrier sensing and enabling/disabling RTS/CTS mechanism $[4,20]$ for a purpose of performance planning.

\section{A. WLAN MAC Important Functions Overview}

Referring to MAC protocols, there are two particular functions configurable cumulatively with RTS frames operation depending on the application services traffic to manage. These are distributed coordination function (DCF) and point coordination function (PCF) [4, 20, 18, 21]. They can be assisted by other sub-system's functions such as. queuing disciplines (QD), enhanced distributed channel access (EDCA); and hybrid controlled channel access / hybrid 
coordinated function (HCCA / HCF) Their configurations along with RTS/CTS mechanism can help deal with the issue of resource contention between WLAN communicating stations [42]. Figure 6 shows how their functional operation compares and complement each other.

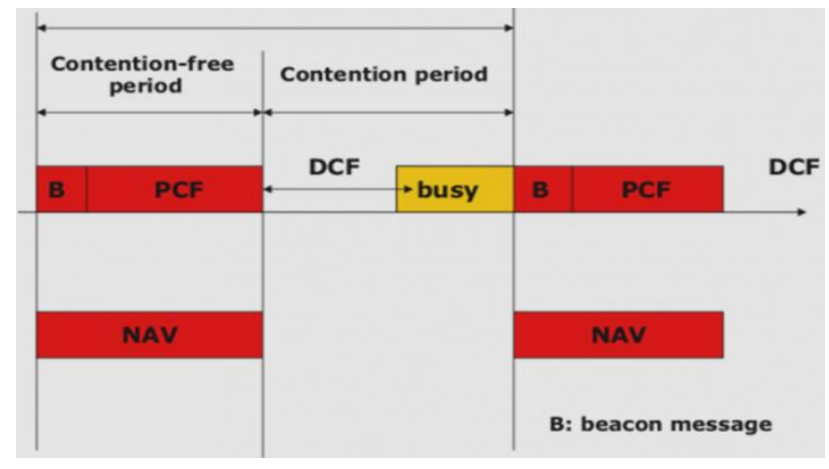

Fig. 6. Comparing DCF vs. PCF operation

According to [16], DCF (if implemented alone) will lead to many collisions at peak periods. Thus, DCF and PCF are genereally configured together for any of applicable schemes in the framework. However, PCF is seen as more useful than DCF; it assists DCF and it particularly enables provisioning WLAN QoS under IEEE 802.11e standard. In fact, PCF let create a model of QoS solution convenient for real-time multimedia applications. Also, [13] noticed that most of designed protocols to overcome hidden and exposed node problems made use of DCF in turn supported by RTS/CTS mechanism.

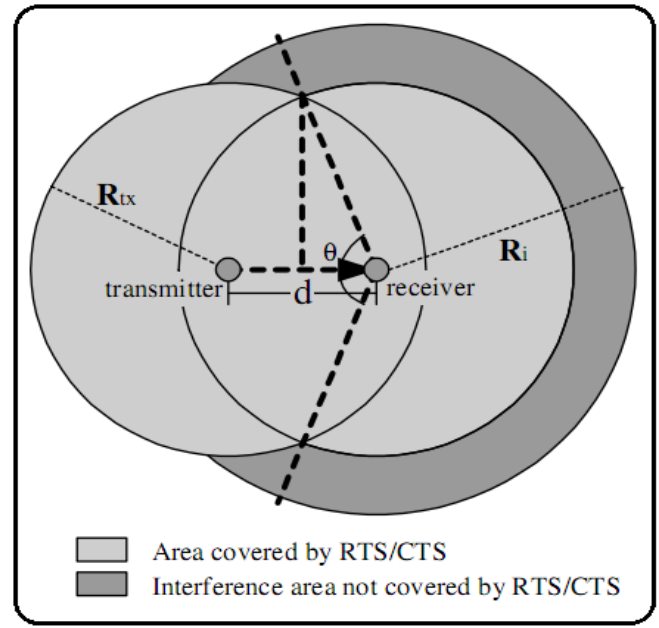

Fig. 7. RTS/CTS handshake effectiveness estimation -i.e. for ('d') larger than $0.56 * \mathrm{R}_{\mathrm{tx}}$ and smaller than $\mathrm{R}_{\mathrm{tx}}[4]$

\section{B. RTS/CTS Framework Mathematical Modeling/Design}

Like any other technologies, IEEE 802.11's RTS/CTS handshake got some limitations as compared to its theoretically expected performance [4].

For instance, this mechanism is not able to fully eliminate the hidden terminal problems. Anyhow, as example, here is an introduction to a mathematical modeling related to some direct parameters that are linked to this complex problem regarding WLAN performance.
1) Modeling the space between hidden nodes for communications

The model of problem on hidden station involved two general elements: the relative location and position of nodes to their local AP and their respective range of transmission power to each other and to the AP position (Figure 7 illustration). All these have in common a distance between the two nodes relatively to an intersection of their (RTS/CTS) radio range coverage compared to the non-covered area.

Based on Figure 7, there are three radio ranges labeled as $\mathrm{R}_{\mathrm{tx}}$ : transmission range; $\mathrm{R}_{\mathrm{tx}}$ : carrier sensing range and $\mathrm{R}_{\mathrm{i}}$ : interference range. According [3]'s authors, the conditions on the distance "d" to satisfy the receiving node's signal power (i.e. on the hidden node) must obey the law of the following equation:

$$
P_{r}=P_{t} G_{t} G_{r} \frac{h_{t}^{2} h_{r}^{2}}{d^{4}}
$$

with:

- $P_{t}$ the transmission power; $G_{t}$ and $G_{r}$ respectively the antenna gains of transmitter and receiver; $h_{t}$ and $h_{r}$ the height of both antennas; $d$ the distance between the transmitter and the receiver.

\section{2) Receiving Station Estimate Signal To Noise Ratio}

The demonstration in Figure 7 is with an assumption of being in a homogeneous MANET environment. Therefore a signal arriving at the receiver is considered to be valid if the signal to noise ratio (SNR) is above a certain threshold (SNR_THRESHOLD). Then, SNR is given by $\mathrm{SNR}=\mathrm{Pr} / \mathrm{Pi}$; and considering homogeneous radios, (SNR) is computed with the equation (2).

$$
\begin{gathered}
\text { SNR }=P_{r} / P_{i}=\left(\frac{r}{d}\right)^{4} \geq S N R \_ \text {THRESHOLD } \\
\text { where } r \geq \sqrt[4]{S N R \_ \text {THRESHOLD }} * d \quad \text { [Eq.2]. }
\end{gathered}
$$

This means that to successfully receive a signal, the interfering nodes must be:

$$
r \geq \sqrt[4]{\text { SNR }- \text { THRESHOLD }} * d \text { (meters) }
$$

away from the receiver. In fact, in practice, SNR_THRESHOLD is usually set to 10 . Thus, $\mathrm{R}_{\mathrm{i}}$ is as in [Eq.3]:

$$
R_{i}=\sqrt[4]{10} * d=1.78 * d[\mathrm{Eq} 3]
$$

Based on equation (3), when the transmitter- receiver distance " $\mathrm{d}$ " is larger than $\mathrm{R}_{\mathrm{tx}} / 1.78=0.56 * \mathrm{R}_{\mathrm{tx}}\left(\mathrm{R}_{\mathrm{tx}}\right.$ being the transmission range), the interference range then exceeds the transmission range. This is easy to understand that power level needed for interrupting a transmission is much smaller than that of successfully delivering a packet. The interference area around a receiver is defined as $A_{i}=\pi R_{i}^{2}$. And all the nodes within the interference area will be hidden nodes of the considered receiver.

\section{3) Brief Theory For RTS/CTS Use Effectiveness Planning}

Among additional parameters for estimation and prediction in practical control, the effectiveness of RTS/CTS ( $\mathrm{E}_{\mathrm{RTS} / \mathrm{CTS}}$ ) is defined for the following involved elements:

- $\mathrm{Ai}=$ Total interference area. 
- $\mathrm{A}_{\mathrm{iRTS} / \mathrm{CTS}}=$ Interference area where nodes can receive RTS or CTS successfully.

Hence: $\quad \mathrm{E}_{\mathrm{RTS} / \mathrm{CTS}}=\mathrm{A}_{\mathrm{iRTS} / \mathrm{CTS}} / \mathrm{A}_{\mathrm{i} .} \quad[\mathrm{Eq} 4]$.

Then, based on equation (4), for $\left(\mathrm{d}<=0.56 * \mathrm{R}_{\mathrm{tx}}\right)$, apparently $A_{i R T S / C T S}$ is equal to $A_{i}$ since transmission range is larger than the interference range. Thus, ERTS/CTS will be almost equal to 1 . And when "d" (the transmitter's distance to the receiver's antenna, Fig.7) increases beyond $0.56 * \mathrm{R}_{\mathrm{tx}}$, $\mathrm{A}_{\mathrm{iRTS} / \mathrm{CTS}}$ becomes smaller than " $\mathrm{A}$ " resulting in the $\left(\mathrm{E}_{\mathrm{RTS} / \mathrm{CTS}}\right)$ smaller than 1; etc. Further estimation on the RTS/CTS threshold related parameters can be found in [4, 13].

\section{Practical Configurations in Performance Design}

Here are briefly some commonly recommended RTS/RTS threshold values and range that can be configured in wireless networks testing. In finding, a WLAN permanent monitoring is the only better way to find out which "exact" values to make use as a result of good understanding the network behaviour over some specific periods of time (e.g. day times, week days, etc.). In other words, it is generally recommended to determine appropriate periods when RTS/CTS must be enabled or disabled with suitable threshold settings. For, this added touch is practically the easier way to adjust the WLAN topology change due to users' relative moving position within its wireless coverage environment,

According to $[5,20]$ there is a range of RTS/CTS (and fragmentation) threshold setting values that can help network manager to choose from after a routine assessment of the network behaviors. The typical activities for carrying out such assessments are discussed in $[5,33\}]$. As threshold setting examples:

a) When having many users far from the access point, lower the threshold to 2304 bytes; then verify the new outcome;

b) For Fragmentation as solution; default size threshold is 2346 bytes and the standard range is 256-2346 bytes;

c) In real-world, these indicated setting values should be tried between 256 and 2346 until getting the fine tune with the data flow is normalized.

d) Etc.

These above cited examples are among the most common settings in work testing cases from many literatures.

\section{RTS/CTS IN INTEGRATED SCHEMES BASED WLAN PERFORMANCE SOLUTIONS}

RST/CTS mechanism is still implemented nowadays in temporary use manner (i.e. manual configuration vs. automated insertion). In fact, from traditional function, it is considerable as associated tools with any QoS solutions implemented to support the network performance. An observation from most of the research works on this mechanism is much more considered as technique most appropriate to combat transmission collisions at wireless AP $[2,3,4,6]$.

As discussed under above section 3, it is important to have a clear understanding between performance and service quality performance. That is because it helps know and localize where their measurement's parameters intervene/act with respect to the network structural layers. This includes the actual role played by RTS/CTS in the assessment of the two, which are commonly ignored or invisible part of the networks integrated solutions. A comprehensible study on IEEE 802.11 WN/WLAN has been carried out in $[30,31]$ articles about its background, technology standards and applications.

Similar study has been found in related articles reviews and surveys conducted by $[28,24,43,44]$ authors particularly about its (IEEE 802.11) implementation for networks QoS support. For instance, QoS matter is reviewed in some contemporary research articles like [24] with a great attempt of classifying and categorization methods, protocols and methods for network performance and QoS design. Nevertheless, those materials are well-presented, but for the most knowledge people; since all technical details focused only on the latest knowledge or terminologies beyond RTS/CTS in argumentation throughout each articles review. However, another article [45] is an overview of QoS in wireless data networks, which summarized the commonly used tools and technologies, and included the potentials and important role of RTS/CTS for this purpose in WLAN, Hence, the subject of concern in this article is obviously verifiable from such recent survey papers; thanks for these articles contents' quality and coverage.

Furthermore, recent and oncoming trends in networking data communications is the highest interest in using integrated data or multimedia contents, which requires the implementation of QoS integrated solutions (QoS-IS). Based on (Arindam Paul, 1999) [32] 'QoS-IS' is a standards set from Internet Engineering Task Force (IETF) group to support various network traffic classes with different QoS profiles through some network elements. The system generally works fine subject to the resources availability managed by an admissions control system - a switch or router's policy decisions base. Typically, such network system based QoS-IS (provisioning) will involve either or most of the following scheme elements: congestion avoidance/congestion management mechanisms, per-flow-state maintenance tools, traffic shaping and policing; and link efficiency control. Hence the lack of this disposition leads to offering all existing resources to any traffic classes and thus leading the network traffic onto a best-effort support. Some of related activities for QoS-IS are illustrated in above Figure 5.

\section{A. RTS Importance and WLAN QoS Provisioning Techniques}

The foremost use - enabling RTS operation, is to combat any possible packets collision between clients, which have become invisible or insensible one to another. This happens due to the interferences phenomenon, or clients unreachable in their radio range. Such a situation occurs when user's clients are too much wide spread and thus become unheard in the AP wireless medium coverage.

In overall above situations, RTS/CTS are proven capable in WLAN performance support by testing, then minimizing and avoiding collisions $[5,35,38]$. In RTS/CTS operation (Figures $1,2 \& 3$ ) the station initiating a communication process sends RTS frame to the AP. RTS and CTS exchange 
acts as environment free testing process. This in turn enables reducing packets transmission collision. And when properly enabled congestion context and threshold settings correct choice), then collisions can sufficiently be avoided [2, 5, 15]. RTS/CTS can also be considered as a fundamental tool for WLAN performance management [23, 36, 41]. That is because the protocols can successfully handle following two critical issues in performance management: (a) Problem of hidden stations [43]; (b) securing performance troubles with extra-protection that reduces/eliminates the risks of collisions [6]. With this way the delay is minimized and the throughput E2E is guaranteed with less data loss. Overall, RTS/CTS offer concrete implementation that assures high probability of performance degradation avoidance; for, it is linked to above (a\&b) situations [43] than contrary solutions.

Many research studies have discussed WLAN performance issues along with various enhancement solutions. Contemporary trends in networking applications are likely more about mobile networks deployment and multimedia applications as network contents. Meanwhile, this category of application services are much demanding in their service quality performance requirements which are very sensible to collisions phenomenon. And collisions are proven to have severe and intolerable effects on these popular WLAN/WMN service applications (e.g. voice, audio and rich media) [3, 6, $32,43]$ for either of the following reason:

a) Collisions will cause packets loss and thus throughputs decrease.

b) Collision will also introduce additional transmission delays other than the systematic one.

At the overall networks level, (a) and (b) cause congestion between LANs' sections, due to missing frames retransmissions, which introduce in most cases an unrealistic congestion [31].

Other additional performance solutions (beyond the scope of this articles review) are generally used in combination with default RST/CTS for network performance enhancement.

A WLAN performance can be improved after an observation of some persisting decreases in throughputs delivery or excessive E2E delays. Relatively to WLAN performance issues, such facts are the revealing effects of collisions between wireless communicating nodes' transmitted packets $[1-6,13,14,15]$. The additional collision's defects include the data loss and congestions at network level that contribute directly to the performance and QoS degradation.

As simpler and practical solution to these issues, one can turn on (or off) the RTS/CTS protocols on every WLAN clients $[2,6]$; they are recognized as powerful for controlling and minimizing collisions happening [2, 3, 17, 43]. Regarding some of RTS/CTS limitations, [15] led a valuable study on some perspectives and came up with some proposed solutions. Different RTS/CTS schemes as framework exist for this purpose (e.g. Bandwidth reservation, reducing delays and loss). Figure 8, refers to the case for BSS based WLAN.

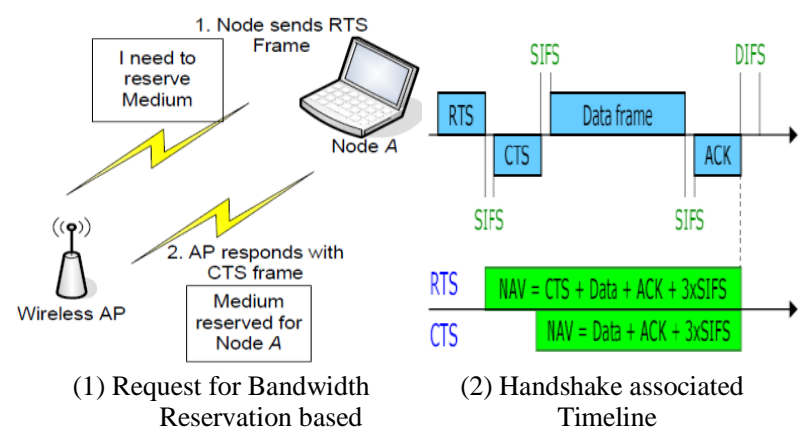

Fig. 8. Bandwidth Reservation request using RTC/CTS mechanism

In this case, the RTS/CTS scheme consists of five frames (Fig. 8(2)); but the bandwidth reservation is ensured actually by two: Short Inter-frame Space (SIFS) and Network Allocation Vector (NAV) [2]. This framework is associated with the called single exclusive access or shared MAC $[3,6$, 15] configurations mode.

However, with distributive MAC (DMAC) known also as multiple accesses MAC, the RTS/CTS framework differs significantly according to the wireless network (WN) deployment's access schemes in use. That is whether it applies a random access or a controlled access techniques [18] to suit the WN deployment. The most important detail is that, DMAC is the scheme appropriate for MANET environment $[6,13,15$, 18 ] in order to provide ad-hoc wireless mobile node's services application with an acceptable QoS level. QoS problems get more complex due to different factors. These include an attempt to accommodate various application services concurrently running (even for those without any performance requirements). Another factors case and the most challenging is about keeping healthy the network state information accuracy, which is merely void [27]. And, this impossibility has a room for a satisfactory solution under WLAN/WN managers' duty; that is about well-monitoring WLAN and using wisely RTS/CTS features in order to prevent or at least minimize the congestion occurrence known up to here as the bottom/root cause of the network poor performance and then QoS degradation.

\section{CONCLUSION}

This paper has discussed about RTS/CTS framework paradigm, and particularly the important role that this mechanism plays along with existing WLAN QoS provisioning methods. A review on heterogeneous networks (e.g. taking multimedia network as a general model) using both early and recent literatures has proven that networks congestion can be considered as the major root for networks performance degradation. In fact, in wireless networks environment, packets transmission collision is technically the primary source of data loss; E2E decrease is the immediate effect. However, RTS/CTS stand as the fundamental mechanism that is suitable and simpler solution to the above discussed WLAN systematic issue. Thanks to WLAN and network technologies evolution, this tool is friendly made accessible on some WLAN routers. 
A particular contribution of this review has been to demonstrate that these basic and simpler tools are kept mute (absent) in most study papers that discussed about WLAN performance / QoS issues. Even though RTS/CTS alone may not provide today's WN model with sufficient QoS support, they remain among the most powerful tools for WLAN managers' support. For, they help those managers in allowing WLAN clients to enjoy typical QoS (if any) as offered by their services provider. Moreover, in addition to the source origin of networks congestion and the growing trends of service applications, new QoS factors are added up. Thus, the following details show that we will not get rid of their causes for soon. They are the technology with endless imperfections and limitations, user's increasing demands for services higher quality/features; etc. Therefore, the end of the stated issues needs to be always considered with their fundamental solution's tools (RTS/CTS) as far as networking communications will still be alive and using IEEE 802.11.

A future work for this articles review will be a practical lab testing on the efficiency of the RTS/CTS mechanism features as QoS support for multimedia based mobile WLANs networks; including their direct insertion into multiple schemes based QoS integrated solutions.

\section{ACKNOWLEDGMENT}

The authors would like to thank the Universiti Teknikal Malaysia (UTeM) through its research and innovations department services (CRIM) for the payment of this journal article registration and publication fees (Footnote extension from first page).

\section{REFERENCES}

[1] Wi-fiplanet.com, Jim Geier, 2002 Improving WLAN Performance with RTS/CTS, Wi-Fi Planet Tutorials; accessed: 25-11-2016, from [http://www.wi-fiplanet.com/tutorials/article.php/1445641/ImprovingWLAN-Performance-with-RTSCTS.htm].

[2] Shakil Akhtar, 2006 Communication Performance of 802.11 WLANs; Proceedings of the 10th WSEAS International Conference on APPLIED MATHEMATICS, Dallas, Texas, USA, November 1-3, 2006 89; accessed: 29-11-2016, from [http://www.wseas.us/elibrary/conferences/2006dallas/papers/519-256.pdf].

[3] Hetal Jasani \& Nasser Alaraje, 2007 Evaluating the Performance of IEEE 802.11 Network using RTS/CTS Mechanism, IEEE EIT 2007 Proceedings; 1-4244-0941-1/07/\$25.00 c 2007 IEEE; retrieved: 10-032016.

[4] Kaixin Xu, Mario Gerla \& Sang Bae, n.d. How effectiveness is RTS/CTS handshake in IEEE 802.11 based ad hoc networks? University of California, Los Angeles, Computer Science Department, Los Angeles, CA 90095, USA; retrieved: 10-03-2016.

[5] INFOSEC, 2014 RTS threshold configuration for improved wireless network performance; accessed: 10-03-2016, at: [http://resources.infosecinstitute.com/rts-threshold-configurationimproved-wireless-network-performance/].

[6] Saikat Ray, Jeffrey B. Carruthers, and David Starobinski, n.d. RTS/CTS-induced congestion in ad hoc wireless LANs (Lecture); Department of Electrical and Computer Engineering --Boston University; accessed: 10-03-2016, at: [www.cacs.louisiana.edu/.../fas9529.p... ].

[7] Jaime Lloret, Miguel Garcia, Hugo Coll and Miguel Edo, 2012 Wireless sensor networks and systems --Wireless Technologies: Concepts, Methodologies, Tools and Applications, DOI: 10.4018/978-1-61350101-6.ch102; Pages: 13; Copyright: @ 2012; retrieved: 29-11-16.

[8] Wireless local area network (WLAN) gateway system; accessed: 22-2210-2016, at: [http://www.google.com/patents/US20130103558].
[9] Lingnan University, 2009 Background and History of Wireless LAN for Lingnan University; Copyright $\odot 2016$ Lingnan University All rights reserved; accessed: 09-09-16, from [https://www.ln.edu.hk/itsc/network/wireless/bg].

[10] Chaim Ziegler, 2009 Wireless LAN Applications, Wireless; retrieved: 11-11-2016; Copyright @ 2009.

[11] Kensuke Miyashita \& ,Yuki Maruno, 2016 Campus Wireless LAN Usage Analysis and Its Applications; Chapter Neural Information Processing; Volume 9947 of the series Lecture Notes in Computer Science pp 563-569; Springer International Publishing; DOI: 10.1007/978-3-319-46687-3_62; Print ISBN: 978-3-319-46686-6; retrieved: 06-12-2016.

[12] USA Information Resources Management Association, 2012 Wireless Technologies: Concepts, Methodologies, Tools and Applications (3 Volumes) Information Resources Management Association (USA) ; _Copyright: (O2012; Pages: 2358, ISBN13: 9781613501016|ISBN10: 1613501013|EISBN13: 9781613501023; DOI: 10.4018/978-1-61350101-6; retrieved: 20-11-16.

[13] Jayasuriya, Sylvie Perreau, Arek Dadej, and Steven Gordon, 2004 Hidden vs. exposed terminal problem in ad hoc networks.; Australian telecommunication networks \& applications conference (ATNAC) 2004 (2004) pp. 52-59; retrieved: 22-07-16.

[14] Khushboo Agarwal and Vikas Sejwar, 2015 Avoidance of hidden terminal \& exposed terminal problem using directional MAC protocol; International Journal of Future Generation Communication and Networking ; Vol. 8, No. 4 (2015), pp. 231-238; ISSN: 2233-7857 IJFGCN Copyright (C) 2015 SERSC; retrieved: 20-07-16.

[15] L. Boroumand, R. H. Khokhar, L. A. Bakhtiar and M. Pourvahab, 2012 A review of techniques to resolve the hidden node problem in wireless networks. Smart Computing Review, vol. 2, no. 2, April 2012; DOI: 10.6029/smartcr.2012.02.001; retrieved: 20-07-16.

[16] Tankonyvtar.Hu/En, Vilmos Simon , 2014 Wireless and mobile technologies for the future internet, article online; accessed: 12-07-16, at [http://www.tankonyvtar.hu/en/tartalom/tamop412A/20110050_09_wire less_mobile_technologies/ar01s03.html].

[17] www.comlab.hut.fi, Teknillinen Korke Akoulu, n.d. Lecture 4: WLAN 2 (MAC layer operation); accessed: 12-07-16, at: [www.comlab.hut.fi/studies/3240/luentokalvot/4_wlan2.ppt].

[18] Andrew Von Nagy , 2011 Understanding Wi-Fi carrier sense; accessed: 28-06-16, [http://www.revolutionwifi.net/revolutionwifi/2011/03/understandingwi-fi-carrier-sense.html].

[19] www.cs.jhu.edu, David Holmer, 2002. Wireless medium access, lecture; retrieved: 12-12-16.

[20] INFOSEC, 2014 RTS threshold configuration for improved wireless network performance; accessed: 28-06-16, at: [http://resources.infosecinstitute.com/rts-threshold-configurationimproved-wireless-network-performance/].

[21] Lecture, Markku Renfors, n.d. IEEE 802.11; (IEEE 802.11 overview, architecture and MAC); accessed: 28-06-16, at: [http://docplayer.net/12481121-802-11-markku-renfors-partly-based-onstudent-presentation-by-lukasz-kondrad-tomasz-augustynowiczjaroslaw-lacki-jakub-jakubiak.html].

[22] E-Computernotes.com, Dinesh Thakur, n.d. What is congestion control? Describe the congestion control algorithm commonly use; accessed: 2806-16, at: Available at: [ecomputernotes.com/computernetworkingnotes/...networks/].

[23] Kensuke Miyashita \& ,Yuki Maruno, 2016 Campus Wireless LAN Usage Analysis and Its Applications; Chapter Neural Information Processing; Volume 9947 of the series Lecture Notes in Computer Science pp.563-569; Springer International Publishing; DOI: 10.1007/978-3-319-46687-3_62; Print ISBN: 978-3-319-46686-6; retrieved: 06-12-2016.

[24] Qiang Ni, Lamia Romdhani and Thierry Turletti, 2004 A survey of QoS enhancements for IEEE 802.11 wireless LAN: research articles; Journal Wireless Communications \& Mobile Computing archive; Volume 4 Issue 5, August 2004, Pages 547 - 566, DOI: 10.1002/wcm.v4:5; John Wiley and Sons Ltd. Chichester, UK; retrieved: 06-12-2016. 
[25] Whatis.techtarget, Margaret Rouse, 2006 What is performance?; Part of the Computing fundamentals glossary: Definition from WhatIs.com; accessed: 27-11-2016 [http://whatis.techtarget.com/definition/performance].

[26] Whatis.techtarget.com, Margaret Rouse2006 What is quality? Part of the Programming glossary: Definition from WhatIs.com; accessed: 2711-2016, from [http://whatis.techtarget.com/definition/quality].

[27] Amandeep Kaur, 2011 An overview of quality of service computer network; Indian Journal of Computer Science and Engineering (IJCSE), Vol. 2 No. 3 Jun-Jul 2011; ISSN: 0976-5166; retrieved: 10-11-2016.

[28] Richard Hill, 2012 Overview of quality of service (QoS); APT-ITU workshop on the International Telecommunica3ons Regulations Bangkok, 6-8 February 2012 Richard Hill, ITU; retrieved: 20-10-2016,

[29] BROADCOM, Philippe Klein, 2008 802.11 QoS overview; IEEE Plenary Meeting - Nov 08 Dallas, TX, avb-phkl-802-11-qos-overview0811-1; retrieved: 20-10-2016.

[30] Ibrahim Al Shourbaji, (n.d.) An Overview of Wireless Local Area Networks (WLAN); Computer Networks Department; Jazan University; Jazan 82822-6649; retrieved: 09-09-2016.

[31] Kevin J. Negus, and Al Petrick, 2008 History of Wireless Local Area Networks (WLANs) in the Unlicensed Bands ; George Mason University Law School Conference, Information Economy Project, Arlington, VA., info, Vol. 11 Iss: 5, pp.36 - 56; DOI: http://dx.doi.org/10.1108/14636690910989324; 09-09-2016.

[32] Arindam Paul, 1999 QoS in Data Networks: Protocols and Standards; accessed: 28-11-2016, from [http://www.cse.wustl.edu/ jain/cis78899/ftp/qos_protocols.pdf].

[33] www.ciscopress.com, 2010 Performance Considerations - WLAN Design: Range, Performance of WLAN; accessed: 18-05-16.; at: [www.ciscopress.com/articles/article.asp?p=1613796...3].

[34] Home-Network-Help.Com,n.d. Improving wireless network performance by tuning advanced wireless settings on wireless device; accessed: 18-05-16.; at [http://www.home-network-help.com/wirelessnetwork-performance.html].

[35] Wang, Kaishun Wu and Mounir Hamdi, 2012 Combating hidden and exposed terminal problems in wireless networks; IEEE Transactions on Wireless Communication, vol.10, no.10, 40-12, 2012; retrieved: 25-1116.
[36] Hui Ma, Eiman Alotaibi, and Sumit Roy, n.d. Analysis and simulation model of physical carrier sensing in IEEE 802.11 mesh networks; retrieved: 25-11-16.

[37] Felix Diaconu, 2012 IEEE 802.11 MAC frame fragmentation performances in jammed environments; Bul. Inst. Polit. Iaşi, t. LVIII (LXII), f. 2, 20121; retrieved: 17-06-16.

[38] Jaime Lloret, Miguel Garcia, Hugo Coll and Miguel Edo, 2012 Wireless sensor networks and systems --Wireless Technologies: Concepts, Methodologies, Tools and Applications, DOI: 10.4018/978-1-61350101-6.ch102; Pages: 13; Copyright: @ 2012. Retrieved: 29-11-16.

[39] Stefan Mangold, Sunghyun Choi, Peter May, Ole Klein, Guido Hiertz \& Lothar Stibor, n.d. IEEE 802.11e wireless LAN for quality of service. Retrieved: 29-11-16.

[40] Mangold, Sunghyun Choi, Guido R. Hiertz, Ole Klein,\& Bernhard Walke, 2003 Analysis of IEEE 802.11e for QoS support in wireless LANs; IEEE wireless communications, volume: 10 , issue: 6 , page(s): 40 - 50; ISSN : 1536-1284 ; INSPEC accession number: 7873965; DOI: 10.1109/MWC.2003.1265851; IEEE communications society. retrieved: 17-06-16.

[41] Namita Yadav \& Sanjay Sachan, 2014 Analysis and the performance effectiveness of RTS /CTS mechanism in IEEE 802.11; International Journal of Emerging Trends \& Technologies in Computer Science (IJETTCS); Volume 3, Issue 4, July-August 2014, ISSN 2278-6856; retrieved: $18-05-16$.

[42] Ashwini Dalvi, Pamukumar Swamy and B B Meshram, 2011 DCF improvement for satisfactory throughput of 802.11 WLAN; International Journal on Computer Science and Engineering (IJCSE);Vol. 3 No. 7 July 2011; ISSN : 0975-3397; retrieved: 18-05-16.

[43] Journal of Engineering Research \& Technology; Volume/Issue: Vol.2 Issue 4 (April - 2013), e-ISSN: 2278-0181; retrieved: 18-05-16.

[44] Hua Zhu, Ming Li, Imrich Chlamtac, and B. Prabhakaran. 2004, A survey of quality of service in IEEE 802.11 networks; IEEE Wireless Communications, volume: 11 , issue: 4 ; page(s): 6 - 14 ; ISSN : 15361284; INSPEC Accession Number: 8086234; DOI: 10.1109/MWC.2004.1325887; IEEE communications society; retrieved: 18-05-16.

[45] Adam Petcher, n.d. QoS in Wireless data networks (Online article); accessed: 11-07-16.; at [http://www.cs.wustl.edu/ jain/cse57406/ftp/wireless_qos/]. 